Original paper

\title{
Increased serum glypican-3 is associated with liver stiffness and hepatic dysfunction in children with biliary atresia
}

\author{
Kanjaporn Sirisomboonlarp', Wanvisa Udomsinprasert ${ }^{1,2}$, Ellie McConachie' ${ }^{1}$, Thamonwan Woraruthai', \\ Yong Poovorawan ${ }^{3}$, Sittisak Honsawek ${ }^{1}$ \\ 'Department of Biochemistry, Osteoarthritis and Musculoskeleton Research Unit, Faculty of Medicine, Chulalongkorn University, \\ King Chulalongkorn Memorial Hospital, Thai Red Cross Society, Bangkok, Thailand \\ 2Department of Biochemistry, Faculty of Pharmacy, Mahidol University, Bangkok, Thailand \\ ${ }^{3}$ Center of Excellence in Clinical Virology, Department of Pediatrics, Faculty of Medicine, Chulalongkorn University, King Chulalongkorn \\ Memorial Hospital, Thai Red Cross Society, Bangkok, Thailand
}

\begin{abstract}
Aim of the study: Biliary atresia (BA) is an uncommon disorder of the liver and bile ducts affecting infants and is characterized by progressive fibrosclerosing obstruction of the extrahepatic biliary tree leading to end-stage liver failure. The purpose of this study was to determine serum glypican-3 (GPC3) levels and liver stiffness in children with BA and the correlation of glypican-3 with clinical parameters.

Material and methods: Seventy-five post-Kasai BA patients and 28 healthy age-matched controls were registered. Serum GPC3 levels were examined by enzyme-linked immunosorbent assay. Liver stiffness measurement was analyzed by transient elastography.

Results: BA patients had significantly greater serum GPC3 and liver stiffness values than controls $(p<0.001)$. Serum GPC3 and liver stiffness values were significantly higher in jaundiced BA patients than in non-jaundiced BA patients $(p<0.001)$. Additionally, serum glypican-3 was associated with liver stiffness and serum total bilirubin $(p<0.001$, respectively).

Conclusions: Elevated serum GPC3 levels were associated with hepatic dysfunction and the severity of BA. As a result, serum GPC3 and liver stiffness might serve as biomarkers reflecting the deterioration of hepatic function and the outcome in post-Kasai BA.
\end{abstract}

Key words: biliary atresia, glypican-3, jaundice, liver stiffness, severity.

\section{Address for correspondence}

Sittisak Honsawek, MD, PhD, Department of Biochemistry, Osteoarthritis and Musculoskeleton Research Unit, Faculty of Medicine, Chulalongkorn University, King Chulalongkorn Memorial Hospital, Thai Red Cross Society, Bangkok, Thailand, phone: +662-256-4482, fax: +662-256-4482, e-mail: sittisak.h@chula.ac.th

\section{Introduction}

Biliary atresia (BA) is a cholestatic liver disease of infants characterized by inflammatory and fibrotic obliteration of the biliary ducts, leading to severe fibrosis and eventually to biliary cirrhosis [1]. The primary treatment is Kasai portoenterostomy (KPE); however, the majority of BA patients still experience the progression of biliary fibrosis towards cirrhosis and ultimately undergo liver transplantation [2]. This effect has driven researchers towards the search for, and de- velopment of, biochemical markers for detecting the early stage of BA, which may be helpful in improving patient outcome and delaying the need for liver transplants. Even though the precise etiology of BA remains obscure, several factors have reportedly been implicated in its pathogenesis including viral infection, autoimmune-mediated bile duct injury, and environmental factors. Interestingly, defects in embryonic morphogenesis and/or progressive inflammatory process are currently considered as among the most pathogenic events in the development and progression of the 
disease [3]. As such, elucidating the molecular mechanisms underlying BA pathology and their relevance to the embryologic development of biliary tract-mediated inflammation is of paramount importance to the discovery of new therapeutic targets. The development of the bile ducts is complex and incompletely understood; nevertheless, it has been well established that biliary development is governed by several important factors in cell signaling - particularly the Wnt cascade $[4,5]$. For this reason, the possible role of molecules in regulating the embryologic morphogenesis of the biliary tract via Wnt signaling is of great interest to many researchers.

Glypican-3 (GPC3), a heparan sulfate proteoglycan, is a member of the glypican family consisting of GPC1 to GPC6. It attaches to the outer surface of the plasma membrane by a glycosyl-phosphatidylinositol anchor [6]. The GPC3 gene, located on chromosome $\mathrm{Xq} 26$, encodes a product that plays a regulatory role in the growth and differentiation of numerous cells through interactions with a variety of growth factors, including Hedgehogs, fibroblast growth factors, and Wnts [7]. It has been documented that GPC3 mRNA and protein are expressed in a tissue-specific manner, predominantly during embryonic tissue development, indicating that GPC3 might have a potential role in morphogenesis [8]. Therefore, it seems plausible that GPC3 may have an immense potential to be a molecular predictor for the embryologic development of the biliary ducts involved in BA etiology. In addition to its possible effect on morphogenesis, mutation in the GPC3 gene has been shown to contribute to Simpsom-Golabi-Behmel syndrome, which is characterized by overgrowth symptoms such as macrosomia and causes cancerous tumors including Wilms tumor, nephroblastoma, and hepatoblastoma [9]. With such a potent effect on hepatoblastoma, overexpression of GPC3 has been shown to promote the growth of hepatocellular carcinoma (HCC) through stimulating the Wnt signaling pathway [6]. Indeed, systemic GPC3 levels have been previously associated with the severity of HCC, showing its diagnostic values as a circulating biomarker for diagnosis of HCC [6].

Although GPC3 has been reported to be related to the development and progression of several types of tumors, especially HCC, there is limited information on the association between GPC3 and clinical outcomes of BA. Accordingly, the purposes of this study were to investigate circulating GPC3 levels in BA patients compared to healthy controls and to determine the plausible association between circulating GPC3 and clinical parameters of post-operative BA patients.

\section{Material and methods}

The study protocol conformed to the ethical standards outlined in the Declaration of Helsinki and was approved by the Ethical Committee on Human Research of the Faculty of Medicine, Chulalongkorn University. All parents of children were fully informed regarding the study protocol and procedures prior to the children entering the study. Written informed consent was obtained from the participants' parents.

\section{Study population}

Seventy-five BA patients ( 36 boys and 39 girls with mean age of $9.5 \pm 0.6$ years) and 28 healthy children (11 boys and 17 girls with mean age of $9.2 \pm 0.4$ years) were registered in this study. None of them had undergone liver transplantation. Healthy controls attending the Well Baby Clinic at King Chulalongkorn Memorial Hospital for vaccination had normal physical findings and no underlying disease. BA patients were classified into two groups according to their serum total bilirubin (TB). According to theirjaundice status, children with BA were divided into a non-jaundice group (TB $<2 \mathrm{mg} / \mathrm{dl}$, $n=35$ ) and a persistent jaundice group ( $\mathrm{TB} \geq 2 \mathrm{mg} / \mathrm{dl}$, $n=40)$. Furthermore, portal hypertension $(\mathrm{PH})$ was assessed by the presence of ascites and/or esophageal varices observed by endoscopy. Thirty-two children had no portal hypertension but the remaining 43 did.

\section{Laboratory methods}

Peripheral venous blood specimens were obtained from every subject, centrifuged, and kept instantly at $-20^{\circ} \mathrm{C}$ until used. Double-blind quantitative measurement of circulating GPC3 levels was assessed using commercially available sandwich enzyme-linked immunosorbent assay (ELISA) (R\&D Systems, Minneapolis, MN, USA). Corresponding to the manufacturer's instruction, recombinant human GPC3 standards and serum samples were pipetted into every well of a microplate, which was precoated with specific antibody to GPC3. After incubating for $2 \mathrm{~h}$ at room temperature, all wells were washed completely 3 times with washing reagent. Subsequently, glypican-3 conjugate was pipetted into every well and incubated for $2 \mathrm{~h}$ at room temperature. After 3 washes, substrate solution was added to each well and then the microplate was incubated for $20 \mathrm{~min}$ at room temperature without light. Ultimately, all reactions were terminated by the stop reagent and the optical density was evaluated with an automated microplate reader at $450 \mathrm{~nm}$. The intensity of color derived is clearly proportionate to the quantity 
Table 1. Demographic data, biochemical characteristics, and liver stiffness scores of biliary atresia patients and healthy controls

\begin{tabular}{lccc}
\hline Variables & $\begin{array}{c}\text { BA } \\
(\boldsymbol{n}=\mathbf{7 5})\end{array}$ & $\begin{array}{c}\text { Controls } \\
(\boldsymbol{n}=\mathbf{2 8})\end{array}$ & $\boldsymbol{p}$-value \\
\hline Age (years) & $9.5 \pm 0.6$ & $9.2 \pm 0.4$ & 0.4 \\
\hline Gender (female : male) & $39: 36$ & $17: 11$ & 0.3 \\
\hline Albumin (g/dl) & $4.2 \pm 0.1$ & - & $\mathrm{NA}$ \\
\hline Total bilirubin (mg/dl) & $2.6 \pm 0.4$ & - & $\mathrm{NA}$ \\
\hline Direct bilirubin (mg/dl) & $2.2 \pm 0.4$ & - & $\mathrm{NA}$ \\
\hline AST (IU/l) & $146.6 \pm 13.2$ & $26.4 \pm 0.8$ & $<0.001$ \\
\hline ALT (IU/l) & $138.9 \pm 12.5$ & $9.1 \pm 0.7$ & $<0.001$ \\
\hline ALP (IU/l) & $438.2 \pm 35.8$ & - & $\mathrm{NA}$ \\
\hline Liver stiffness (kPa) & $28.8 \pm 2.9$ & $4.0 \pm 0.2$ & $<0.001$ \\
\hline Glypican-3 (ng/ml) & $8.7 \pm 0.1$ & $6.5 \pm 0.3$ & $<0.001$ \\
\hline
\end{tabular}

Data are expressed as mean $\pm S E M$. BA - biliary atresia, AST - aspartate aminotransferase, ALT - alanine aminotransferase, ALP - alkaline phosphatase, NA - not applicable

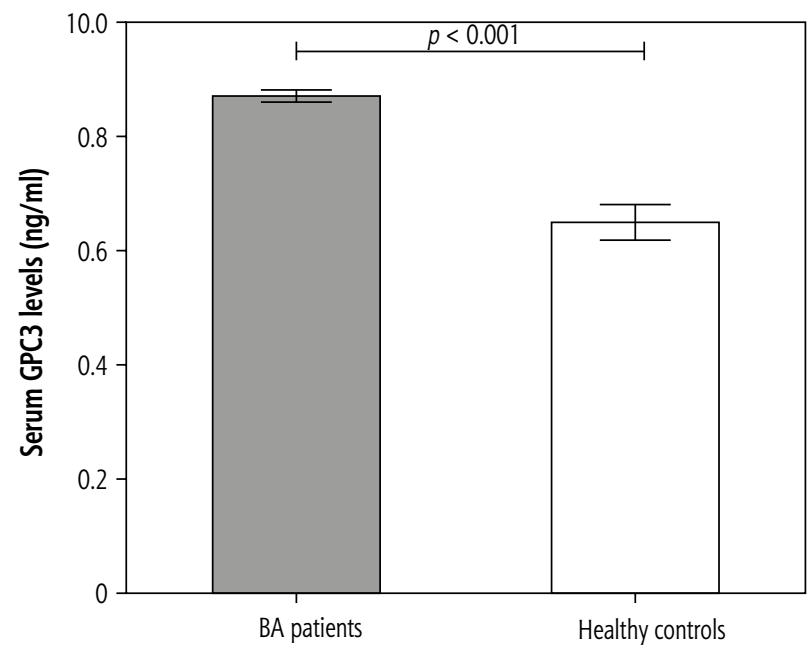

Fig. 1. Comparison of serum glypican-3 (GPC3) levels in postoperative biliary atresia (BA) patients and healthy controls. Data are expressed as mean \pm SEM

of GPC3 in the samples. A standard optical densityconcentration curve was created for determination of the GPC3 value. The liver function tests including serum albumin, TB, direct bilirubin (DB), aspartate aminotransferase (AST), alanine aminotransferase (ALT), and alkaline phosphatase (ALP) were measured using a Hitachi 912 (Roche Diagnostics, Basel, Switzerland) automated machine at the central laboratory of our hospital.

\section{Liver stiffness measurement}

Transient elastography (FibroScan, Echosens, Paris, France) was used to measure the liver stiffness between 25 and $65 \mathrm{~mm}$ from the skin surface, which is approx- imately equivalent to the volume of a cylinder $1 \mathrm{~cm}$ in diameter and $4 \mathrm{~cm}$ in length. The measurements were performed by placing a transducer probe of FibroScan on the intercostal space at the area of the right lobe of the liver with patients lying in a dorsal decubitus position with maximum abduction of the right arm. The target location for measurement was a liver portion that was at least $6 \mathrm{~cm}$ thick, and devoid of major vascular structures. The measurements were performed until 10 validated results had been obtained with a success rate of at least $80 \%$. The median value of the 10 validated scores was considered the elastic modulus of the liver, and it was expressed in kilopascals $(\mathrm{kPa})$.

\section{Statistical analysis}

Statistical analysis was performed using the Statistical Package for the Social Sciences (SPSS) Version 22.0 (IBM Corporation, Somers, NY, United States). Comparisons of demographic and clinical parameters between groups were performed using the chi-square and Student's unpaired $t$-test when appropriate. Correlation between numerical data was acquired using Pearson's correlation coefficient $(r)$. Data were expressed as mean \pm standard error of the mean (SEM). All the $p$-values $<0.05$ based on a two-tailed test were considered statistically significant.

\section{Results}

\section{Comparison between biliary atresia patients and healthy controls}

Seventy-five post-Kasai BA subjects and 28 ageand gender-matched unaffected controls were prospectively registered in the current study. The baseline characteristics of BA patients and control subjects are presented in Table 1. There was no significant difference in age or gender between BA and control groups. However, circulating GPC3 values were substantially higher in BA subjects than in healthy controls $(8.7 \pm 0.1$ vs. $6.5 \pm 0.3 \mathrm{ng} / \mathrm{ml}, p<0.001$ ) (Fig. 1). Additionally, BA subjects had considerably higher liver stiffness values than control volunteers $(28.8 \pm 2.9$ vs. $4.0 \pm 0.2 \mathrm{kPa}$, $p<0.001)$.

\section{Comparison between jaundiced group and non- jaundiced group of biliary atresia patients}

Biliary atresia patients were categorized into the jaundiced group $(n=40)$ and non-jaundiced group $(n=35)$. The clinical data and biochemical features 
of patients according to jaundice status are illustrated in Table 2. Children with BA with jaundice exhibited significantly higher circulating GPC3 values than children with BA without jaundice $(9.1 \pm 0.2$ vs. $8.2 \pm 0.1$ $\mathrm{ng} / \mathrm{ml}, p<0.001$ ) (Fig. 2). Moreover, mean liver stiffness measurement of the BA group with jaundice was substantially elevated compared with that of the BA group without jaundice $(41.6 \pm 4.1$ vs. $14.2 \pm 2.3 \mathrm{kPa}$, $p<0.001)$.

Further analysis showed that circulating GPC3 levels were more elevated in children with $\mathrm{BA}$ with $\mathrm{PH}$ than those without $\mathrm{PH}(9.2 \pm 0.2$ vs $8.0 \pm 0.1 \mathrm{ng} / \mathrm{ml}$, $p<0.001$ ) (Fig. 3). Moreover, circulating GPC3 was positively correlated with serum AST $(r=0.367$, $p=0.001)$, ALT $(r=0.320, p=0.005)$, TB $(r=0.555$, $p<0.001)$, and liver stiffness measurement $(r=0.455$, $p<0.001$ ) in BA patients (Fig. 4).

\section{Discussion}

Given that a younger age when undergoing KPE is considered a predictor of improved survival of native liver and overall survival of BA, a timely diagnosis of BA appears to be the most effective way to improve the patient outcome following KPE [10]. To date, there are still no reliable non-invasive biomarkers for discriminating BA infants from other forms of neonatal cholestatic jaundice. Regarding this, development of sensitive and robust circulating biomarkers for early detection of BA is needed. It seems clear that a better understanding of the molecular mechanisms behind BA pathology is of critical importance to the discovery of a potential indicator for predicting disease progression. Although the exact cause of BA remains a matter of debate, several factors, particularly molecule-mediated inflammation, have been reportedly involved in its pathogenesis [11-13]. In the present study, we investigated circulating GPC3 levels obtained from BA patients compared to healthy controls and associations between circulating levels of GPC3 and clinical parameters of hepatic dysfunction in BA patients.

It is well recognized that GPC3, a membrane-bound heparan sulfate proteoglycan, acts as a co-receptor regulating the signaling activity of numerous growth factors, such as bone morphogenic protein 7 , fibroblast growth factor 2 , and Wnt $[7,14]$. The $\mathrm{Wnt} / \beta$-catenin signaling pathway has been suggested to play a potential role in regulation of biliary tract development [15], thereby establishing the possible influence of GPC3-mediated Wnt/ $\beta$-catenin signaling in BA etiology. Physiologically, the biological action of GPC3 has been shown to be responsible for hepatic pathology in chronic liver disease - especially HCC [16-18].
Table 2. Comparison of biliary atresia patients without and with jaundice

\begin{tabular}{lccc}
\hline Variables & $\begin{array}{c}\text { BA patients } \\
\text { with jaundice } \\
(\boldsymbol{n}=\mathbf{4 0})\end{array}$ & $\begin{array}{c}\text { BA patients } \\
\text { without jaundice } \\
(\boldsymbol{n}=\mathbf{3 5})\end{array}$ & $\boldsymbol{p}$-value \\
\hline Age (years) & $9.4 \pm 0.6$ & $9.7 \pm 0.5$ & 0.4 \\
\hline Gender (female : male) & $21: 19$ & $18: 17$ & 0.5 \\
\hline Albumin (g/dl) & $3.9 \pm 0.2$ & $4.4 \pm 0.1$ & 0.008 \\
\hline Total bilirubin (mg/dl) & $4.4 \pm 0.6$ & $0.7 \pm 0.1$ & $<0.001$ \\
\hline Direct bilirubin (mg/dl) & $3.8 \pm 0.6$ & $0.4 \pm 0.1$ & $<0.001$ \\
\hline AST (IU/l) & $202.7 \pm 18.9$ & $82.5 \pm 10.8$ & $<0.001$ \\
\hline ALT (IU/l) & $185.2 \pm 18.2$ & $86.1 \pm 11.9$ & $<0.001$ \\
\hline ALP (IU/l) & $538.4 \pm 46.5$ & $330.7 \pm 49.0$ & 0.002 \\
\hline Liver stiffness (kPa) & $41.6 \pm 4.1$ & $14.2 \pm 2.3$ & $<0.001$ \\
\hline Glypican-3 $(\mathrm{ng} / \mathrm{ml})$ & $9.1 \pm 0.2$ & $8.2 \pm 0.1$ & $<0.001$ \\
\hline
\end{tabular}

Data are expressed as mean $\pm S E M$. BA - biliary atresia, AST - aspartate aminotransferase, ALT - alanine aminotransferase, ALP - alkaline phosphatase

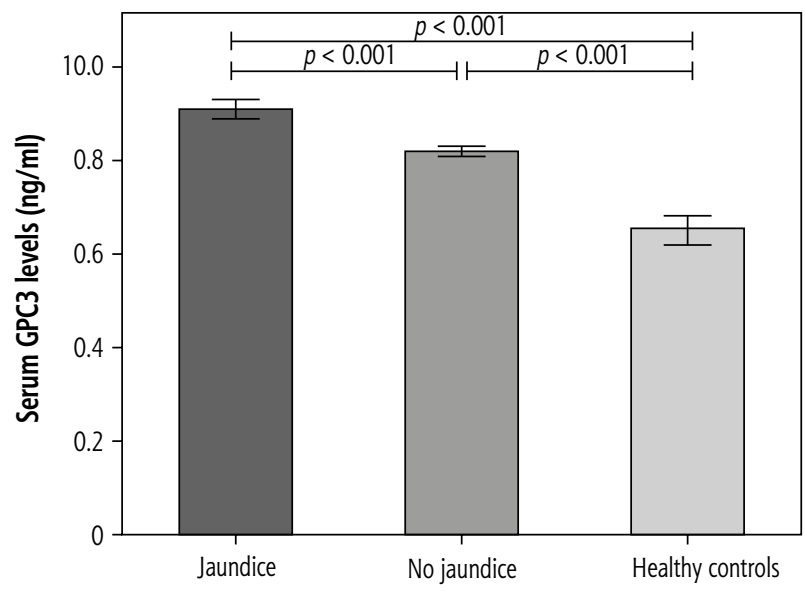

Fig. 2. Comparison of serum glypican-3 (GPC3) levels in biliary atresia (BA) patients with jaundice, BA patients without jaundice, and controls. Data are expressed as mean \pm SEM

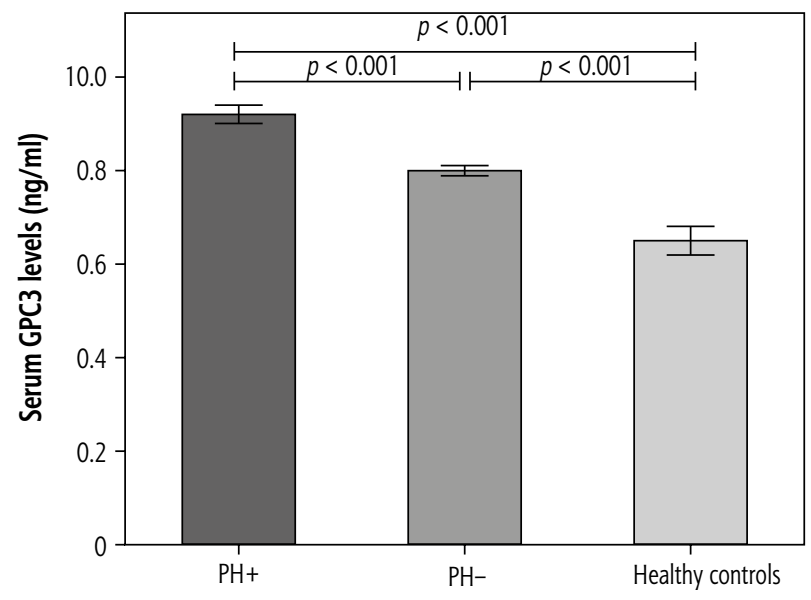

Fig. 3. Comparison of serum glypican-3 (GPC3) levels in biliary atresia (BA) patients without portal hypertension (PH), patients with $\mathrm{PH}$, and controls. Data are expressed as mean \pm SEM 

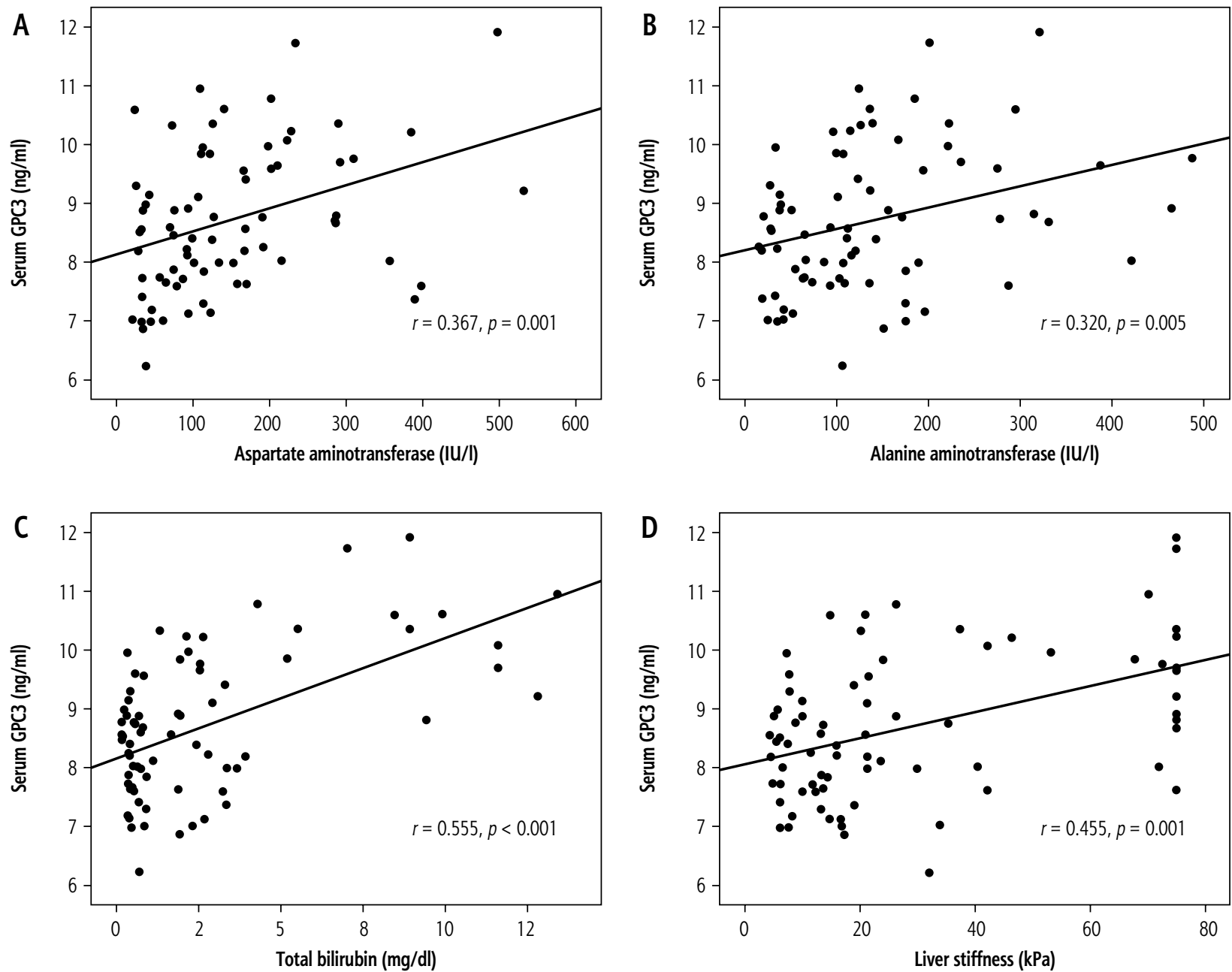

Fig. 4. Scatter diagram and correlation analysis in biliary atresia patients. Serum glypican-3 (GPC3) levels are correlated with (A) serum aspartate aminotransferase, (B) serum alanine aminotransferase, (C) serum total bilirubin, (D) liver stiffness

Indeed, Capurro and colleagues investigated the effect of GPC3 on HCC cell lines and found that GPC3 stimulates the growth of HCC through the activation of canonical Wnt signaling, making GPC-3-mediated Wnt signaling an attractive therapy [19]. Notably, as GPC3 can be released into the circulation from the cell surface by Notum, a lipase that cleaves glycosyl-phosphatidylinositol (GPI) anchors [20,21], it may have diagnostic values as a circulating marker for human diseases. Interestingly, a recent study demonstrated that circulating GPC3 levels were significantly higher in patients with HCC than in both patients with other liver diseases and healthy controls [22]. Furthermore, expression of GPC 3 mRNA in the circulation has been reportedly associated with TNM stage, periportal cancerous embolus, and extrahepatic metastasis in patients with HCC [23]. These findings are in agreement with our study showing that circulating GPC3 levels are considerably higher in BA patients than in healthy controls. Further analysis revealed that children with advanced BA including jaundice and portal hypertension had substantially higher circulating GPC3 levels than those in earlier stages. Additionally, elevated circulating GPC3 levels were positively associated with AST, ALT, TB, and liver stiffness value in BA patients. Collectively, our findings indicate that GPC3 may play a possible role in the mechanism of hepatocellular damage and liver fibrogenesis in postoperative BA patients, and it could be involved in the severity of bile duct obliteration. It is therefore reasonable to speculate that circulating GPC3 might be developed as a non-invasive biomarker identifying the progression of BA disease. This hypothesis is supported by Kandil and colleagues' study [24] showing that up-regulation of circulating GPC3 mRNA was a more sensitive and specific biomarker for monitoring metastasis of HCC. The authors also note the diagnostic utility of GPC 3 in the specimens to aid in discriminating metastatic tumors of HCC from benign liver lesions. In the light of our considerations, it is apparent that the possibility of 
GPC3 having a regulatory role in hepatic injury is currently of great interest for the development of GPC 3 as a biomarker for hepatic dysfunction of post-operative BA patients.

However, the mechanisms behind the connection of elevated circulating levels of GPC 3 and BA pathology have yet to be elucidated. It is tempting to postulate that an increase in circulating levels of GPC3 in BA patients, notably in those with a poor outcome, could be attributable to an imbalance between GPC3 synthesis and its clearance. In this context, the elevation of circulating GPC3 levels may be involved in a defensive response by the body to fight against hepatocellular injury and further hepatic fibrogenesis. In addition, a reduction in GPC3 clearance may result from the destruction of biliary ducts, a feature unique to BA compared to the control, which in turn would increase levels of GPC3 in BA serum. Alternatively, apart from the liver, other tissues including the skin and the brain, known to be important sources of GPC3, might produce and secrete GPC3 into the circulation.

It should be emphasized, however, that we are aware of some inherent limitations regarding the current study. First, this study was designed as a cross-sectional study, which does not allow the determination of cause and effect relationships. It is recommended that prospective cohort or experimental studies be performed to elucidate the exact association of GPC3 levels with the pathogenesis of BA. Second, only circulating GPC3 has been analyzed. Further immunohistochemical studies of GPC3 in the liver could yield more valuable information on the pathogenic role of GPC3 in BA. Given that BA is a sporadic disease, the sample size of BA subgroups and healthy controls in this study was relatively small. For this reason, future observations with larger samples of BA patients and healthy controls are needed to reach an unequivocal conclusion.

In conclusion, the current study is the first to demonstrate increased circulating GPC3 levels in BA patients, particularly in advanced BA patients with jaundice and portal hypertension. Moreover, circulating GPC3 levels were positively associated with jaundice status, liver dysfunction, and the degree of fibrosis in post-operative BA. Circulating GPC 3 with prognostic potential has previously been reported in a variety of pathological contexts, especially HCC [25], and these findings indicate that circulating GPC3 may serve as a plausible non-invasive biomarker reflecting the disease severity in post-Kasai BA. However, future validation with prospective studies is required to elucidate the utility of circulating GPC3 levels as a biomarker in larger populations, and in combination with other biochemical markers, in an effort to facilitate early detection and ameliorated patient outcome.

\section{Acknowledgements}

This research has been supported by the Thailand Research Fund (RSA5880019), the Research Chair Grant from the National Science and Technology Development Agency (NSTDA). The authors gratefully acknowledge the Research Core Facility of the Department of Biochemistry and Chulalongkorn Medical Research Center (ChulaMRC) for kindly providing facilities.

\section{Disclosure}

Authors report no conflict of interest.

\section{References}

1. Mack CL. The pathogenesis of biliary atresia: evidence for a virus-induced autoimmune disease. Semin Liver Dis 2007; 27: 233-242.

2. Balistreri WF, Grand R, Hoofnagle JH, et al. Biliary atresia: current concepts and research directions. Summary of a symposium. Hepatology 1996; 23: 1682-1692.

3. Sokol RJ, Mack C. Etiopathogenesis of Biliary Atresia. Semin Liver Dis 2001; 21: 518.

4. Zong Y, Stanger BZ. Molecular mechanisms of bile duct development. Int J Biochem Cell Biol 2011; 43: 257-264.

5. White BD, Chien AJ, Dawson DW. Dysregulation of Wnt/beta-catenin signaling in gastrointestinal cancers. Gastroenterology 2012; 142: 219-232.

6. Filmus J, Capurro M, Rast J. Glypicans. Genome Biol 2008; 9: 224.

7. Song HH, Shi W, Xiang Y, Filmus J. The loss of Glypican-3 induces alterations in Wnt signaling. J Biol Chem 2005; 280: 2116-2125.

8. Song HH, Filmus J. The role of glypicans in mammalian development. Biochim Biophys Acta 2002; 1573: 241-246.

9. Ho M, Kim H. Glypican-3: A new target for cancer immunotherapy. Eur J Cancer 2011; 47: 333-338.

10. Jimenez-Rivera C, Jolin-Dahel KS, Fortinsky KJ, et al. International incidence and outcomes of biliary atresia. J Pediatr Gastroenterol Nutr 2013; 56: 344-354.

11. Klemann C, Schröder A, Dreier A, et al. Interleukin 17, produced by $\gamma \delta \mathrm{T}$ cells, contributes to hepatic inflammation in a mouse model of biliary atresia and is increased in livers of patients. Gastroenterology 2016; 150: 229-241.

12. Santetti D, de Albuquerque Wilasco MI, Dornelles CT, et al. Serum proinflammatory cytokines and nutritional status in pediatric chronic liver disease. World J Gastroenterol 2015; 21 : 8927-8934.

13. Jian $\mathrm{ZH}$, Wang LC, Lin CC, Wang JD. The correlation between plasma cytokine levels in jaundice-free children with biliary atresia. World J Pediatr 2015; 11: 352-357.

14. Midorikawa Y, Ishikawa S, Iwanari H, et al. Glypican-3, overexpressed in hepatocellular carcinoma, modulates FGF2 and BMP-7 signaling. Int J Cancer 2003; 103: 455-465.

15. Decaens T, Godard C, de Reynies A, et al. Stabilization of betacatenin affects mouse embryonic liver growth and hepatoblast fate. Hepatology 2008; 47: 247-258. 
16. Zhang J, Zhang M, Ma H, et al. Overexpression of glypican-3 is a predictor of poor prognosis in hepatocellular carcinoma: An updated meta-analysis. Medicine (Baltimore) 2018; 97: e11130.

17. Dong Z, Yao M, Wang L, et al. Down-regulating glypican-3 expression: molecular-targeted therapy for hepatocellular carcinoma. Mini Rev Med Chem 2014; 14: 1183-1193.

18. Montalbano M, Rastellini C, McGuire JT, et al. Role of Glypican-3 in the growth, migration and invasion of primary hepatocytes isolated from patients with hepatocellular carcinoma. Cell Oncol (Dordr) 2018; 41: 169-184.

19. Capurro MI, Xiang YY, Lobe C, et al. Glypican-3 promotes the growth of hepatocellular carcinoma by stimulating canonical Wnt signaling. Cancer Res 2005; 65: 6245-6254.

20. Traister A, Shi W, Filmus J. Mammalian Notum induces the release of glypicans and other GPI anchored proteins from the cell surface. Biochem J 2008; 410: 503-511.

21. Kreuger J, Perez L, Giraldez AJ, Cohen SM. Opposing activities of Dally-like glypican at high and low levels of Wingless morphogen activity. Dev Cell 2004; 7: 503-512.

22. Chen M, Li G, Yan J, et al. Reevaluation of glypican-3 as a serological marker for hepatocellular carcinoma. Clin Chim Acta 2013; 423: 105-111.

23. Yao M, Yao DF, Bian YZ, et al. Values of circulating GPC-3 mRNA and alpha-fetoprotein in detecting patients with hepatocellular carcinoma. Hepatobiliary Pancreat Dis Int 2013; 12 : 171-179.

24. Kandil D, Leiman G, Allegretta M, et al. Glypican-3 immunocytochemistry in liver fine-needle aspirates: a novel stain to assist in the differentiation of benign and malignant liver lesions. Cancer 2007; 111: 316-322.

25. Wang L, Yao M, Pan LH, et al. Glypican-3 is a biomarker and a therapeutic target of hepatocellular carcinoma. Hepatobiliary Pancreat Dis Int 2015; 14: 361-366. 